\title{
ON A NONEQUILIBRIUM THERMODYNAMICS OF CAPILLARITY AND PHASE*
}

\author{
BY \\ MORTON E. GURTIN \\ Carnegie Mellon University, Pittsburgh, PA
}

1. Introduction. It is the purpose of this paper to develop a nonequilibrium thermodynamics for phase transitions, capillarity, and other phenomena involving large concentration gradients. The main ideas are best explained in terms of a binary mixture undergoing isothermal diffusion, and it is within this setting that we frame the theory. The generalization to multiple species is obvious; the extension to nonisothermal behavior will be the subject of a future paper.

We begin with a discussion of the classical theory of diffusion for a mixture in a region $\Omega$. The basic physical quantities, defined for all $\mathbf{x}$ in $\Omega$ and all time $t$, are the concentration $c(\mathbf{x}, t)$, the mass flux $\mathbf{h}(\mathbf{x}, t)$, the mass supply $q(\mathbf{x}, t)$, the free energy $\psi(\mathbf{x}, t)$, and the chemical potential $\mu(\mathbf{x}, t)$; and the underlying laws, for any subregion $P$ of $\Omega$, are balance of mass

$$
\frac{d}{d t} \int_{P} c=-\int_{\partial P} \mathbf{h} \cdot \mathbf{n}+\int_{P} q
$$

and the second law, which for isothermal diffusion has the form

$$
\frac{d}{d t} \int_{P} \psi \leq-\int_{\partial P} \mu \mathbf{h} \cdot \mathbf{n}+\int_{P} \mu q
$$

where $\mathbf{n}$ is the outward unit normal to $\partial P$.

The term

$$
-\int_{\partial P} \mu \mathbf{h} \cdot \mathbf{n}
$$

represents energy carried across $\partial P$ by the diffusing material; this particular form for the energy flux insures that energy flows across $\partial P$ when and only when mass flows across $\partial P$. In a transition region between phases, or in any other region of high capillarity, surface effects not modeled by the classical theory become important, and I believe that to characterize such phenomena one must allow for a flow of energy over and above that carried by the diffusing material.

To help motivate the primitive concepts of my theory, it is instructive to consider the classical model of Gibbs [1] in which a phase interface is represented by a surface - of zero thickness. When the model of Gibbs is considered within a dynamical framework one finds, for $P$ an arbitrary subregion, a flow of energy into $P$ due to

*Received March 24, 1988. 
the motion of $s$. Indeed, since $s$ has energy, energy is carried into and out of $P$ as portions of $s$ enter and leave $P .{ }^{1}$ This flow is clearly not included in the diffusive term (3). There is also a flow of energy into $P$ along $s$ due to the flow of mechanical energy within $s$, a flow present when the material is anisotropic; roughly speaking, this flow compensates for the change in surface energy resulting from rotations of $s .^{2}$ Both of these energy flows are linear ${ }^{3}$ in the normal velocity of $s$.

In the theory presented here a phase interface appears as a thin transition zone $\mathscr{T}$, and an additional energy flux is introduced to represent energy flows of the type discussed above. Here we are faced with a major problem: the transition zone and corresponding normal velocity are not easily identified. A possible means of characterizing $\mathscr{T}$ is with the aid of a potential $\pi(\mathbf{x}, t)$; roughly speaking, $\mathscr{T}$ might correspond to a thin region in which $\pi(\mathbf{x}, t)$ varies rapidly with values in a spinodal-type set between phases, the "normal velocity" of $\mathscr{T}$ being related to the derivative $\dot{\pi}(\mathbf{x}, t)=\partial_{t} \pi(\mathbf{x}, t)$. With this in mind, I add an energy flux of the form

$$
\int_{\partial P} \dot{\pi} \boldsymbol{\zeta} \cdot \mathbf{n}
$$

with $\pi$ a scalar field called the capillarity potential and $\zeta$ a vector field called the capillarity flux. One might visualize (4) as a flow of energy necessary to sustain the transition zone, its nonequilibrium nature clear from the presence of the rate $\dot{\pi}$.

This flux of energy ${ }^{4}$ is one of the two essential ingredients of the theory. The second ingredient is a set of constitutive equations which includes a dependence on higher gradients, ${ }^{5}$ a dependence needed to model situations involving thin regions of rapidly varying concentration.

We therefore add to our list of primitive concepts the capillarity potential $\pi(\mathbf{x}, t)$ and the capillarity flux $\boldsymbol{\zeta}(\mathbf{x}, t)$, and consider constitutive equations involving a dependence on $c$ as well as its higher gradients: ${ }^{6}$

$$
\begin{aligned}
& \psi=\hat{\psi}\left(c, \nabla c, \nabla^{2} c, \nabla^{3} c, \ldots\right), \quad \mu=\hat{\mu}\left(c, \nabla c, \nabla^{2} c, \nabla^{3} c, \ldots\right), \\
& \mathbf{h}=\hat{\mathbf{h}}\left(c, \nabla c, \nabla^{2} c, \nabla^{3} c, \ldots\right), \quad \pi=\hat{\pi}\left(c, \nabla c, \nabla^{2} c, \nabla^{3} c, \ldots\right), \\
& \boldsymbol{\zeta}=\hat{\boldsymbol{\zeta}}\left(c, \nabla c, \nabla^{2} c, \nabla^{3} c, \ldots\right) .
\end{aligned}
$$

Further, we find it useful to define an additional field, the reduced capillarity vector

$$
\boldsymbol{\xi}=\hat{\boldsymbol{\xi}}\left(c, \nabla c, \nabla^{2} c, \nabla^{3} c, \ldots\right),
$$

through $^{7}$

$$
\hat{\boldsymbol{\xi}}=\partial_{c} \hat{\pi} \hat{\zeta}
$$

${ }^{1}$ Cf. Fernandez-Diaz and Williams [13], Gurtin [14].

${ }^{2}$ Gurtin [25], extending to dynamics ideas of Cahn and Hoffman [10, 11].

${ }^{3}$ Cf. Gurtin [21], Eq. (5.6) and [25], Eqs. (2.2), (2.3), and (3.10).

${ }^{4}$ (For a deforming body without diffusion) energy flows-not included in the classical flows of heat and mechanical power-are introduced and systematically treated by Dunn and Serrin [15].

${ }^{5}$ The use of higher gradients to model capillarity is due to van der Waals [2] and Korteweg [3], and more recently to Cahn and Hilliard [4] and Landau and Lifschitz [5]. See also Widom [12].

${ }^{6}$ Here $\nabla^{p}$ denotes the $p$ th gradient, so that $\nabla^{2}$ is not the Laplacian; we write $\Delta$ for the Laplacian and div for the divergence.

${ }^{7}$ We write $\partial_{A} f$ for the (generally partial) derivative of the function $f$ with respect to the variable $A$. In particular, for $f$ scalar-valued and $\mathbf{A}$ a tensor of order $n, \partial_{\mathbf{A}} f$ has components $\partial f / \partial A_{i j \cdots k}$. Similarly, $\partial_{A B} f=\partial_{A}\left(\partial_{B} f\right)$, etc. 
A dependence of free energy on concentration gradients is often used to characterize capillarity; within this context, higher gradients appear as a stabilizing influence. Under fairly weak assumptions (cf. the free-energy hypothesis) consistent with this observation, we show, as a consequence of the second law, that the free energy can depend at most on $c$ and the first gradient

$$
\mathbf{g}=\nabla c,
$$

the chemical potential at most on $c, \mathbf{g}$, and the second gradient

$$
\mathbf{G}=\nabla^{2} c \text {. }
$$

In fact, we show that:

(i) the constitutive equations must have the reduced form

$$
\begin{array}{lll}
\psi=\hat{\psi}(c, \mathbf{g}), & \mu=\hat{\mu}(c, \mathbf{g}, \mathbf{G}), \\
\pi=\hat{\pi}(c), & \boldsymbol{\xi}=\hat{\boldsymbol{\xi}}(c, \mathbf{g}) ;
\end{array}
$$

(ii) the free energy generates the chemical potential and reduced capillarity vector through the relations

$$
\begin{aligned}
& \hat{\boldsymbol{\mu}}=\partial_{c} \hat{\psi}-\operatorname{div} \hat{\boldsymbol{\xi}}, \\
& \hat{\boldsymbol{\xi}}=\partial_{\mathbf{g}} \hat{\psi} ;
\end{aligned}
$$

(iii) the mass flux obeys the inequality

$$
\hat{\mathbf{h}} \cdot \nabla \hat{\mu} \leq 0 .
$$

We also consider a quasi-linear theory in which the chemical potential, mass flux, and reduced capillarity vector are linear (affine) functions of the gradients of $c$. We show, as consequences of our general results, that such constitutive equations necessarily have the specific form: ${ }^{8}$

$$
\begin{aligned}
& \psi=\psi_{0}(c)+\frac{1}{2} \mathbf{g} \cdot \mathbf{A g}, \\
& \mu=\psi_{0}^{\prime}(c)-\mathbf{A} \cdot \mathbf{G}, \\
& \boldsymbol{\xi}=\mathbf{A g}, \\
& \mathbf{h}=-\mathbf{K}(c) \nabla \mu,
\end{aligned}
$$

where $\mathbf{A}$ and $\mathbf{K}(c)$ are second-order tensors with $\mathbf{A}$ symmetric, $\mathbf{A} \geq 0, \mathbf{A} \neq 0, \mathbf{K}(c) \geq$ 0 . Here $\psi_{0}(c)$ represents the coarse-grain free energy (the free energy at constant concentration), and $\psi_{0}^{\prime}(c)=d \psi_{0}(c) / d c$.

The constitutive equations (5), when combined with the local form of balance of mass, yield a single partial differential equation for the concentration,

$$
\dot{c}=\operatorname{div}\left\{\mathbf{K}(c) \nabla\left[\psi_{0}^{\prime}(c)-\mathbf{A} \cdot \nabla^{2} c\right]\right\}+q,
$$

which, for an isotropic material with $\mathbf{K}$ constant, reduces to the Cahn-Hilliard equation, ${ }^{9}$

$$
\dot{c}=k \Delta\left[\psi_{0}^{\prime}(c)-a \Delta c\right]+q .
$$

\footnotetext{
${ }^{8}$ We write $\mathbf{u} \cdot \mathbf{v}$ for the inner product of $\mathbf{u}$ and $\mathbf{v}$, regardless of the inner-product space in question. For $\mathbf{A}$ and $\mathbf{B}$ tensors of order $n, \mathbf{A} \cdot \mathbf{B}=A_{i j \cdots k} B_{i j \cdots k}$. (We use components and summation convention where convenient.) For $\mathbf{T}$ an appropriate linear transformation, $\mathbf{T} \geq \mathbf{0}$ signifies that $\mathbf{T}$ is positive semi-definite. ${ }^{9} \mathrm{Cahn}[7,8]$; see also Cahn and Hilliard [9]. The derivation of this equation, within a continuumthermodynamical framework, was a motivating factor for the present study.
} 
We discuss appropriate initial/boundary-value problems for (6), and deduce associated Liapunov functions. Because of the underlying thermodynamics, the latter is not difficult: the Liapunov function for an isolated boundary is the total free energy of $\Omega$.

In a future paper we will relate the present theory to that of $[21,25]$ in which the interface is modeled as a surface of zero thickness.

II. Generalization of the second law. Capillarity flux and capillarity potential. Constitutive equations.

1. Balance of mass. The second law. We now make precise the general discussion of the Introduction. We assume that $\Omega$ is a compact region in $\mathbf{R}^{3}$. Then the primitive physical quantities, defined for all $\mathbf{x}$ in $\Omega$ and all time $t$, are

concentration $c(\mathbf{x}, t)$,

mass flux $\mathbf{h}(\mathbf{x}, t)$,

mass supply $q(\mathbf{x}, t)$,

free energy $\psi(\mathbf{x}, t)$,

chemical potential $\mu(\mathbf{x}, t)$,

capillarity potential $\pi(\mathbf{x}, t)$,

capillarity flux $\zeta(x, t)$;

the basic physical laws, for any subregion $P$ of $\Omega$, are balance of mass

$$
\frac{d}{d t} \int_{P} c=-\int_{\partial P} \mathbf{h} \cdot \mathbf{n}+\int_{P} q
$$

and the second law

$$
\frac{d}{d t} \int_{P} \psi \leq-\int_{\partial P}(\mu \mathbf{h}-\dot{\pi} \boldsymbol{\zeta}) \cdot \mathbf{n}+\int_{P} \mu q,
$$

where $\mathbf{n}$ is the outward unit normal to $\partial P$. Since $P$ is arbitrary, (1.1) and (1.2) have the equivalent local forms

$$
\begin{aligned}
\dot{c} & =-\operatorname{div} \mathbf{h}+q, \\
\dot{\psi} & \leq-\operatorname{div}(\mu \mathbf{h}-\dot{\pi} \boldsymbol{\zeta})+\mu q .
\end{aligned}
$$

and together yield the dissipation inequality

$$
\dot{\psi}-\mu \dot{c}-\operatorname{div}(\dot{\pi} \boldsymbol{\zeta})+\mathbf{h} \cdot \nabla \mu \leq 0 .
$$

The integral laws (1.1) and (1.2) have several simple but important consequences, depending on the behavior of the boundary. We will consider two types of boundary conditions:

(i) isolated boundary:

$$
\boldsymbol{\zeta} \cdot \mathbf{n}=0 \quad \text { and } \quad \mathbf{h} \cdot \mathbf{n}=0 \text { on } \partial \Omega
$$

(ii) uniform boundary:

$$
\mu=\mu_{b} \quad \text { and } \quad \pi=\pi_{b} \text { on } \partial \Omega,
$$

with $\mu_{b}$ and $\pi_{b}$ constant.

The next theorem will yield Liapunov functions for the underlying boundary-value problems. 
Growth TheOREM. Let $q=0$. Then balance of mass and the second law imply that:

(i) for an isolated boundary

$$
\begin{aligned}
& \frac{d}{d t} \int_{\Omega} c=0 \\
& \frac{d}{d t} \int_{\Omega} \psi \leq 0
\end{aligned}
$$

(ii) for a uniform boundary

$$
\frac{d}{d t} \int_{\Omega}\left(\psi-\mu_{b} c\right) \leq 0
$$

2. Constitutive equations. As constitutive equations for free energy, chemical potential, and mass flux we write

$$
\begin{aligned}
\psi & =\hat{\psi}\left(c, \nabla c, \nabla^{2} c, \nabla^{3} c, \ldots\right), \\
\mu & =\hat{\mu}\left(c, \nabla c, \nabla^{2} c, \nabla^{3} c, \ldots\right), \\
\mathbf{h} & =\hat{\mathbf{h}}\left(c, \nabla c, \nabla^{2} c, \nabla^{3} c, \ldots\right),
\end{aligned}
$$

and add similar relations for the capillarity vector and capillarity potential:

$$
\pi=\hat{\pi}\left(c, \nabla c, \nabla^{2} c, \nabla^{3} c, \ldots\right), \quad \zeta=\hat{\zeta}\left(c, \nabla c, \nabla^{2} c, \nabla^{3} c, \ldots\right) .
$$

Let

$$
\begin{aligned}
& S_{n}=\text { space of symmetric } n \text {th order tensors, } n \geq 2, \\
& S_{0}=\mathbf{R}, \\
& S_{1}=\mathbf{R}^{3} .
\end{aligned}
$$

We assume that the response functions $\hat{\psi}, \hat{\mu}, \hat{\mathbf{h}}, \hat{\pi}$, and $\hat{\zeta}$ are smooth on the set $(0,1) \times S_{1} \times S_{2} \times S_{3} \times \cdots$.

Note that each concentration field $c(\mathbf{x}, t)$ generates, through the constitutive equations (2.1) and (2.2), a constitutive process $\mu=(c, \psi, \mu, \mathbf{h}, \pi, \zeta)$; balance of mass $(1.3)_{1}$ then tells us the mass supply ${ }^{10} q$ necessary to support $\rho$, but there is no such freedom in the second law $(1.3)_{2}$, and it seems reasonable to expect that $(1.3)_{2}$-or equivalently (1.4)-will hold in all processes only if certain restrictions are placed on the response functions.

We now make this observation precise. By a concentration field we mean a smooth function $c: \Omega \times \mathbf{R} \rightarrow(0,1)$; and by a constitutive process we mean an array $\mu=$ $(c, \psi, \mu, \mathbf{h}, \pi, \zeta)$ with $c$ a concentration field and $\psi, \mu, \mathbf{h}, \pi$, and $\zeta$ defined on $\Omega \times$ $\mathbf{R}$ through the constitutive equations (2.1) and (2.2). We say that the constitutive equations are compatible with thermodynamics if each constitutive process obeys the dissipation inequality (1.4).

Since the constitutive equations (2.1) and (2.2) depend on gradients of $c$ higher than the first, some additional notation is useful. Let $\nabla^{0} c=c$. Given a function $\hat{\Phi}\left(c, \nabla c, \nabla^{2} c, \nabla^{3} c, \ldots\right)$, we write $D_{p} \hat{\Phi}$ and $D_{p p} \hat{\Phi}$ for the first and second partial derivatives of $\hat{\Phi}$ with respect to the argument $\nabla^{p} c$ :

$$
D_{p} \hat{\boldsymbol{\Phi}}=\partial_{\nabla p_{c}} \hat{\boldsymbol{\Phi}}, \quad D_{p p} \hat{\boldsymbol{\Phi}}=\partial_{\nabla^{p_{c}}} \partial_{\nabla p_{c}} \hat{\boldsymbol{\Phi}}
$$

${ }^{10}$ The inclusion of the supply $q$, which is essential to the logical development of the theory, is due to Serrin [18]. 
Note that for $\hat{\Phi}$ scalar-valued, $D_{p} \hat{\Phi}$ has values in $S_{p}$.

We say that $\hat{\Phi}$ has finite grade if there is an integer $m \geq 0$ such that $\hat{\Phi}\left(c, \nabla c, \nabla^{2} c\right.$, $\left.\nabla^{3} c, \ldots\right)$ is independent of $\nabla^{n} c$ for $n>m$; the smallest such $m$ is then called the grade of $\hat{\boldsymbol{\Phi}}$, written

\section{Grade $\hat{\Phi}$}

and the domain of $\hat{\Phi}$ is identified with the set $\Sigma=(0,1) \times S_{1} \times S_{2} \times S_{3} \times \cdots \times S_{m}$. We say that $\hat{\boldsymbol{\Phi}}$ is strongly of grade $m$, and write

$$
\text { Grade } \hat{\Phi}=m \text { (strongly), }
$$

if Grade $\hat{\Phi}=m$ and if there is no open subset of $\Sigma$ on which $D_{m} \hat{\Phi}$ vanishes.

We henceforth assume that the response functions $\hat{\psi}, \hat{\mu}, \hat{\mathbf{h}}, \hat{\pi}$, and $\hat{\zeta}$ are of finite grade.

For $\hat{\Phi}$ a scalar response function of grade $m$ and $c$ a concentration field, let

$$
\Phi(\mathbf{x})=\hat{\Phi}\left(c(\mathbf{x}), \nabla c(\mathbf{x}), \nabla^{2} c(\mathbf{x}), \ldots, \nabla^{m} c(\mathbf{x})\right),
$$

where, for convenience, we have suppressed the argument $t$. Then, using obvious notation,

$$
\nabla \Phi=\sum_{p=1}^{m} D_{p} \hat{\Phi}\left(c, \nabla c, \nabla^{2} c, \ldots, \nabla^{m} c\right) \nabla^{p+1} c .
$$

The right side of (2.3) defines a function on $(0,1) \times S_{1} \times S_{2} \times S_{3} \times \cdots \times S_{m+1}$; we write $\nabla \hat{\Phi}$ for this function:

$$
(\nabla \hat{\Phi})\left(c, \nabla c, \nabla^{2} c, \ldots, \nabla^{m+1} c\right)=\text { right side of }(2.3) .
$$

An analogous definition applies to

$$
(\operatorname{div} \hat{\Phi})\left(c, \nabla c, \nabla^{2} c, \ldots, \nabla^{m+1} c\right)
$$

for $\hat{\Phi}$ tensor-valued. Note that $\nabla \hat{\Phi}$ is always of grade $m+1$, while $\operatorname{div} \hat{\Phi}$ is generally, but not always, of grade $m+1$.

Finally, we write $\hat{\boldsymbol{\Phi}}(c, 0)$ for the value of $\hat{\boldsymbol{\Phi}}\left(c, \nabla c, \nabla^{2} c, \ldots, \nabla^{m} c\right)$ when all of the concentration gradients vanish:

$$
\hat{\boldsymbol{\Phi}}(c, \mathbf{0})=\hat{\boldsymbol{\Phi}}(c, \mathbf{0}, \mathbf{0}, \ldots, \mathbf{0}) .
$$

III. Consequences of the second law.

3. The compatibility theorem. Capillarity, generally a stabilizing influence, is often characterized by a dependence of free energy on concentration gradients. For that reason we restrict our attention to materials with Grade $\hat{\psi} \geq 1$ (cf. Remark 5). Further, we shall assume that the dependence of $\hat{\psi}$ on the highest gradient, $\nabla^{m} c$, is (in a very weak sense) stabilizing, at least near equilibrium. These assumptions are stated precisely as the free energy hypothesis:

(FH) (i) $\hat{\psi}$ is strongly of grade $m \geq 1$;

(ii) for each $c$,

$$
D_{m} \hat{\psi}(c, \mathbf{0})=0, \quad D_{m m} \hat{\psi}(c, \mathbf{0}) \geq 0, \quad D_{m m} \hat{\psi}(c, \mathbf{0}) \neq 0 .
$$


For convenience, we define a new constitutive variable, the reduced capillarity vector

$$
\boldsymbol{\xi}=\hat{\boldsymbol{\xi}}(c, \mathbf{d}), \quad \mathbf{d}=\left(\nabla c, \nabla^{2} c, \nabla^{3} c, \ldots\right)
$$

through

$$
\hat{\boldsymbol{\xi}}(c, \mathbf{d})=\partial_{c} \hat{\pi}(c, \mathbf{d}) \hat{\boldsymbol{\zeta}}(c, \mathbf{d}) .
$$

Also, since many of our results involve only the first few gradients of $c$, we shall consistently write

$$
\mathbf{g}=\nabla c, \quad \mathbf{G}=\nabla^{2} c, \quad \mathbf{H}=\nabla^{3} c .
$$

Compatibility Theorem. Granted (FH), the constitutive equations are compatible with thermodynamics if and only if the following three conditions are satisfied:

(i) the constitutive equations have the form

$$
\begin{aligned}
& \psi=\hat{\psi}(c, \mathbf{g}), \quad \mu=\hat{\mu}(c, \mathbf{g}, \mathbf{G}), \\
& \pi=\hat{\pi}(c), \quad \boldsymbol{\xi}=\hat{\boldsymbol{\xi}}(c, \mathbf{g}) ;
\end{aligned}
$$

(ii) the free energy generates the chemical potential and reduced capillarity vector through the relations

$$
\begin{aligned}
& \hat{\mu}=\partial_{c} \hat{\psi}-\operatorname{div} \hat{\boldsymbol{\xi}}, \\
& \hat{\boldsymbol{\xi}}=\partial_{\mathbf{g}} \hat{\psi} ;
\end{aligned}
$$

(iii) $\partial_{c} \hat{\pi}$ never vanishes;

(iv) the mass flux is consistent with the inequality

$$
\hat{\mathbf{h}} \cdot \nabla \hat{\mu} \leq 0 .
$$

The proof of this theorem is given in Section 5.

Remark 1. Compatibility with thermodynamics and $(\mathbf{F H})$ actually require that

$$
\begin{array}{llrl}
\text { Grade } \hat{\psi} & =1 \text { (strongly), } & & \text { Grade } \hat{\pi}=0 \text { (strongly), } \\
\text { Grade } \hat{\mu}=2, & & \text { Grade } \hat{\zeta}=\text { Grade } \hat{\xi}=1 .
\end{array}
$$

Remark 2. By (3.3) and (3.4), we can rewrite the constitutive equation for the chemical potential in the alternative form

$$
\hat{\mu}(c, \mathbf{g}, \mathbf{G})=\partial_{c} \hat{\psi}(c, \mathbf{g})-\partial_{c \mathbf{g}} \hat{\psi}(c, \mathbf{g}) \cdot \mathbf{g}-\partial_{\mathbf{g g}} \hat{\psi}(c, \mathbf{g}) \cdot \mathbf{G} .
$$

Remark 3. The restrictions (3.3) and (3.4) do not generally follow if the hypothesis (FH) is dropped. Indeed, the constitutive equations

$$
\begin{aligned}
\psi & =\psi_{0}(c)+(\boldsymbol{\zeta} \otimes \mathbf{A}) \cdot \mathbf{H}, \\
\mu & =\psi_{0}^{\prime}(c), \\
\pi & =\mathbf{A} \cdot \mathbf{G}, \\
\boldsymbol{\zeta} & =\text { constant } \\
\mathbf{h} & =-\nabla \mu
\end{aligned}
$$

are compatible with thermodynamics, but not with (3.3), (3.4), or (FH). 
Remark 4. Assume that the constitutive equations are compatible with thermodynamics. Consider a second constitutive theory, also compatible with thermodynamics, in which

$$
\hat{\pi} \text { and } \hat{\zeta} \text { are replaced by response functions } \pi^{*} \text { and } \zeta^{*} \text {. }
$$

Balance of mass (1.1) does not involve the fields $\pi$ and $\zeta$, while the second law (1.2) involves them only through the combination $\dot{\pi} \zeta$. Thus the transformation (3.6) will leave these laws unaltered if, given any concentration field $c$,

$$
\hat{\pi}(c) \cdot \hat{\zeta}(c, \mathbf{g})=\pi^{*}(c) \cdot \zeta^{*}(c, \mathbf{g})
$$

on $\Omega \times \mathbf{R}$; in this case we will refer to (3.6) as thermodynamically invariant. Trivially, transformation of capillarity variables is thermodynamically invariant if and only if the transformation leaves the reduced capillarity vector unchanged. In fact, the transformation defined by

$$
\pi^{*}(c)=c, \quad \boldsymbol{\zeta}^{*}(c, \mathbf{g})=\hat{\boldsymbol{\xi}}(c, \mathbf{g})
$$

for all $c$ and $\mathbf{g}$ is thermodynamically invariant. Thus: replacing the capillarity vector by the reduced capillarity vector and the capillarity potential by the concentration is a thermodynamically invariant transformation. In view of this result, we may, without loss of generality, replace the capillarity term in the second law (1.2) by

$$
\int_{\partial P} \dot{c} \boldsymbol{\xi} \cdot \mathbf{n} \text {. }
$$

Further, it is clear from the Compatibility Theorem that $\hat{\xi}$ is uniquely determined by the free energy, while $\hat{\pi}$ and $\hat{\zeta}$ are not. Indeed, given any function $\tilde{\pi}$ with nonvanishing derivative on $(0,1)$, replacing $\hat{\pi}$ and $\hat{\zeta}$ by $\tilde{\pi}$ and $\left(\tilde{\pi}^{\prime}\right)^{-1} \hat{\xi}$ is thermodynamically invariant, and this defines the complete set of thermodynamically invariant transformations.

Remark 5. If $(\mathbf{F H})$ is replaced by the assumption Grade $\hat{\psi}=0$, then compatibility with thermodynamics leads to the restrictions $\hat{\mu}=\partial_{c} \hat{\psi}, \hat{\boldsymbol{\xi}}=\mathbf{0}$, and to the conclusion that replacing $\hat{\pi}$ with 0 and $\hat{\zeta}$ with 0 is a thermodynamically invariant transformation. Thus, as would be expected, for Grade $\hat{\psi}=0$ the theory is classical.

4. Algebraic preliminaries to the proof of the compatibility theorem. Recall that $S_{0}=\mathbf{R}, S_{1}=\mathbb{R}^{3}$, and $S_{n}(n \geq 2)$ is the space of symmetric $n$th order tensors. Recall also that the inner product of two $n$th order tensors $\mathbf{A}$ and $\mathbf{B}$ is defined by $\mathbf{A} \cdot \mathbf{B}=A_{i j \cdots k} B_{i j \cdots k}$.

Given a tensor $\mathbf{A}$ of order $n \geq 2$, we write $\operatorname{sym} \mathbf{A}$ for its symmetric part: $\operatorname{sym} \mathbf{A}$ is the unique tensor in $S_{n}$ with the property:

$$
(\operatorname{sym} \mathbf{A}) \cdot \mathbf{B}=\mathbf{A} \cdot \mathbf{B} \quad \text { for all } \mathbf{B} \in S_{n} .
$$

We write $\mathbf{A} \otimes \mathbf{B}$ for the tensor product of tensors $\mathbf{A}$ and $\mathbf{B}$ :

$$
(\mathbf{A} \otimes \mathbf{B})_{i j \cdots k m n \cdots r}=A_{i j \cdots k} B_{m n \cdots r} .
$$

For $\mathbf{A} \in S_{n}$ and $\mathbf{B} \in S_{p}, \mathbf{A} \otimes \mathbf{B}$ will usually arise as the linear transformation from $S_{p}$ into $S_{n}$ defined by

$$
(\mathbf{A} \otimes \mathbf{B}) \mathbf{C}=(\mathbf{B} \cdot \mathbf{C}) \mathbf{A}
$$


for all $\mathbf{C} \in S_{p}$. For $\mathbf{v}$ a vector, we write $\otimes^{n} \mathbf{v}$ for the tensor product of $\mathbf{v}$ with itself $n$ times $\left(\left(\otimes^{n} \mathbf{v}\right) \in S_{n}\right)$.

The next lemma is crucial to our proof of the compatibility theorem.

LEMMA 1. Let $n \geq 1$ be an integer.

(i) Let $\Psi: S_{n} \rightarrow S_{k}$ be linear and consistent with

$$
\left(\otimes^{k} \mathbf{a}\right) \cdot \Psi\left(\otimes^{n} \mathbf{v}\right)=0
$$

for all vectors $\mathbf{a}$ and $\mathbf{v}$. Then

$$
\boldsymbol{\Psi}=\mathbf{0} .
$$

(ii) Let $\mathbf{v} \in S_{1}, \Psi \in S_{n}$. Then

$$
\operatorname{sym}(\mathbf{v} \otimes \Psi)=\mathbf{0} \quad \text { implies } \quad \mathbf{v} \otimes \Psi=\mathbf{0} .
$$

(iii) Let $\Psi: S_{n} \rightarrow S_{n}$ be linear and symmetric and suppose that $\Psi \geq 0, \Psi \neq 0$. Then (considering $\Psi$ as a tensor of order $2 n$ )

$$
\operatorname{sym} \Psi \neq 0 .
$$

Proof. (i) This is a trivial generalization of the standard result: if $\phi\left(\mathbf{v}_{1}, \mathbf{v}_{2}, \ldots, \mathbf{v}_{k}\right)$ is a symmetric $k$-linear form on $\mathbb{R}^{3}$, then

$$
\phi(\mathbf{v}, \mathbf{v}, \ldots, \mathbf{v})=0 \text { for all } \mathbf{v} \in \mathbf{R}^{3} \text { implies } \phi=0 .
$$

To verify (i) choose $\otimes^{n} \mathbf{a}$ arbitrarily and consider the symmetric $k$-linear form

$$
\phi\left(\mathbf{v}_{1}, \mathbf{v}_{2}, \ldots, \mathbf{v}_{k}\right)=\left(\otimes^{n} \mathbf{a}\right) \cdot \Psi\left(\operatorname{sym}\left(\mathbf{v}_{1} \otimes \mathbf{v}_{2} \otimes \cdots \otimes \mathbf{v}_{k}\right)\right) .
$$

By (4.1), this form vanishes when the $\mathbf{v}_{i}$ coincide, and hence, by (4.3), must vanish in general. Thus, writing $\Psi^{\mathrm{T}}: S_{k} \rightarrow S_{n}$ for the transpose of $\Psi$, and using the fact that the tensors $\operatorname{sym}\left(\mathbf{v}_{1} \otimes \mathbf{v}_{2} \otimes \cdots \otimes \mathbf{v}_{k}\right)$ span $S_{k}$, we are led to the conclusion that

$$
\mathbf{A} \cdot \boldsymbol{\Psi}^{\mathrm{T}}\left(\otimes^{n} \mathbf{a}\right)=0 \quad \text { for all } \mathbf{A} \in S_{k} \quad \text { and } \mathbf{a} \in \mathbf{R}^{3} \text {. }
$$

Thus, the argument applied above yields

$$
\mathbf{A} \cdot \mathbf{\Psi}^{\mathrm{T}}\left(\operatorname{sym}\left(\mathbf{a}_{1} \otimes \mathbf{a}_{2} \otimes \cdots \otimes \mathbf{a}_{n}\right)\right)=0,
$$

and $\boldsymbol{\Psi}=\mathbf{0}$.

(ii) Assume that $\operatorname{sym}(\mathbf{v} \otimes \Psi)=\mathbf{0}$, or equivalently that

$$
(\mathbf{v} \otimes \Psi) \cdot \mathbf{B}=\mathbf{0} \text { for all } \mathbf{B} \in S_{n+1} .
$$

We will show that $\mathbf{v} \neq \mathbf{0}$ implies $\Psi=\mathbf{0}$. Assume that $\mathbf{v} \neq \mathbf{0}$ and take $\mathbf{B}=\otimes^{n+1} \mathbf{w}$, which leads to the conclusion that $\Psi \cdot\left(\otimes^{n} \mathbf{w}\right)=0$ for all $\mathbf{w}$ not perpendicular to $\mathbf{v}$, and hence, by continuity, for all $w$. The desired conclusion therefore follows from (4.3).

(iii) Assume $\operatorname{sym} \Psi=0$, so that

$$
\left(\otimes^{n} \mathbf{v}\right) \cdot \Psi\left(\otimes^{n} \mathbf{v}\right)=0
$$

for all vectors $\mathbf{v}$. Thus, since $\Psi$ is symmetric and $\geq 0$,

$$
\left(\otimes^{n} \mathbf{a}\right) \cdot \Psi\left(\otimes^{n} \mathbf{v}\right)=0
$$

for all vectors a and $\mathbf{v}$. Thus, by (i), $\Psi=\mathbf{0}$, a contradiction. 
5. Proof of the Compatibility Theorem. The proof that (i)-(iv) of the Compatibility Theorem imply compatibility with thermodynamics is straightforward and can safely be omitted. We therefore confine our attention to the converse assertion.

Assume that the constitutive equations are compatible with thermodynamics.

The verification of (i)-(iv) will consist of a series of lemmas. The first lemma is central to the Coleman-Noll [6] procedure for finding thermodynamic restrictions on constitutive equations; roughly speaking, this lemma asserts that we can arbitrarily specify $c$ and $\dot{c}$ at any given time, and that we can arbitrarily specify the spatial gradients of $c$ and $\dot{c}$ of any order at any given point and time.

VARIATION Lemma. Choose $t_{0} \in \mathbb{R}$.

(i) Let $c_{0}(\mathbf{x})$ and $v_{0}(\mathbf{x})$ be smooth functions on $\Omega$, and suppose that $c_{0}(\mathbf{x})$ has values in $(0,1)$. Then there is a concentration field $c(\mathbf{x}, t)$ such that

$$
c\left(\mathbf{x}, t_{0}\right)=c_{0}(\mathbf{x}) \text { and } \dot{c}\left(\mathbf{x}, t_{0}\right)=v_{0}(\mathbf{x}) \text { for all } x \in \Omega .
$$

(ii) Choose $\mathbf{x}_{0} \in \Omega$ and let $N$ be a given integer. Then given $\mathbf{B}_{n}, \mathbf{C}_{n} \in S_{n}$ for $n=0,1, \ldots, N$ with $\mathbf{B}_{0} \in(0,1)$ it is possible to find a concentration field $c(\mathbf{x}, t)$ such that

$$
\nabla^{n} c\left(\mathbf{x}_{0}, t_{0}\right)=\mathbf{B}_{n} \quad \text { and } \quad \nabla^{n} \dot{c}\left(\mathbf{x}_{0}, t_{0}\right)=\mathbf{C}_{n} \text { for } n=0,1, \ldots, N \text {. }
$$

Proof (Lemma). (i) Since $\Omega$ is compact and $c_{0}, v_{0}$ smooth, $c_{0}$ has values in a compact subset of $(0,1)$, while $v_{0}$ has values in a compact subset of $\mathbb{R}$. Thus we can find a smooth function $\beta(t)$ on $\mathbb{R}$ such that $\dot{\beta}\left(t_{0}\right)=1$ and such that the function defined on $\Omega \times \mathbb{R}$ by $c(\mathbf{x}, t)=c_{0}(\mathbf{x})+\beta(t) v_{0}(\mathbf{x})$ has values in $(0,1)$. The function $c$ is the desired concentration field.

(ii) Using polynomials of degree $N$, it is a trivial matter to construct functions $f(\mathbf{x})$ and $v_{0}(\mathbf{x})$ such that

$$
\nabla^{n} f\left(\mathbf{x}_{0}\right)=\mathbf{B}_{n} \quad \text { and } \quad \nabla^{n} v_{0}\left(\mathbf{x}_{0}\right)=\mathbf{C}_{n} \quad \text { for } n=0,1, \ldots, N .
$$

Let $\phi(\mathbf{x})$ be a smooth function with compact support in $\Omega$ such that $\phi \equiv 1$ in some neighborhood of $\mathbf{x}_{0}$ and such that $\phi f$ has values in $(0,1)$ (which is possible since $\left.\mathbf{B}_{0} \in(0,1)\right)$. Then (i) with $c_{0}=\phi f$ and $v_{0}$ yields a concentration field $c$ with the desired properties.

The next lemma generalizes a classical result of Gibbs.

LEMMA 2. Constitutive processes satisfy the Gibbs relation

$$
\dot{\psi}=\mu \dot{c}+\operatorname{div}(\dot{\pi} \zeta)
$$

and the inequality

$$
\mathbf{h} \cdot \nabla \mu \leq 0
$$

Proof. Choose an arbitrary constitutive process (with $c$ the corresponding concentration field), an arbitrary time $t_{0}$, and a scalar $\alpha$. By (i) of the Variation Lemma, there is a second concentration field $b$ such that, for all $\mathbf{x} \in \Omega, b\left(\mathbf{x}, t_{0}\right)=c\left(\mathbf{x}, t_{0}\right)$, but $\dot{b}\left(\mathbf{x}, t_{0}\right)=\alpha \dot{c}\left(\mathbf{x}, t_{0}\right)$. If we apply the dissipation inequality at $t_{0}$ to the constitutive process generated by $b$, and use the fact that $\alpha$ and $t_{0}$ are arbitrary, we are led to (5.1) and (5.2). 
The next lemma provides a new method of obtaining restrictions on constitutive equations; its proof is based on the standard variational technique of obtaining EulerLagrange equations. Here we use the following notation: for $\mathbf{A}(\mathbf{x})$ a tensor of order $n$ at each $\mathbf{x}, \operatorname{div} \mathbf{A}(\mathbf{x})$ is the tensor of order $n-1$ defined by

$$
[\operatorname{div} \mathbf{A}(\mathbf{x})]_{j k \cdots p}=\partial_{x_{i}} A_{i j k \cdots p}(\mathbf{x}) .
$$

(Note that contraction is over the first subscript.) Also, recall that, by (FH),

$$
m:=\text { Grade } \hat{\psi} \geq 1 \text {. }
$$

LEMMA 3.

$$
\hat{\mu}=\partial_{c} \hat{\psi}+\sum(-1)^{p} \operatorname{div}^{p}\left(D_{p} \hat{\psi}\right),
$$

the sum being from $p=1$ to $p=m$.

Proof. Let $\varphi(\mathbf{x})$ be a smooth scalar field with compact support in $\Omega$. Then given any smooth field $\mathbf{A}(\mathbf{x})$ on $\Omega$ with values in $S_{p}$,

$$
\int_{\Omega} \mathbf{A} \cdot \nabla^{p} \varphi=(-1)^{p} \int_{\Omega}\left(\operatorname{div}^{p} \mathbf{A}\right) \varphi .
$$

Choose a constitutive process and an arbitrary time $t_{0}$, and let $b(\mathbf{x})$ denote the corresponding concentration field at $t_{0}$. Let $c(\mathbf{x}, t)$ denote the concentration field constructed in the proof of (i) of the Variation Lemma with $c_{0}(\mathbf{x})=b(\mathbf{x})$ and $v_{0}(\mathbf{x})=\varphi(\mathbf{x})$. Since $\varphi$ has compact support in $\Omega$, the capillarity potential for $c(\mathbf{x}, t)$ has a time derivative that vanishes on $\partial \Omega$. Thus the Gibbs relation (5.1), applied at $t=t_{0}$ to the constitutive process generated by $c(\mathbf{x}, t)$, yields, by virtue of $(5.4)$ with $\mathbf{A}=D_{p} \hat{\psi}$,

$$
0=\int_{\Omega}\left\{\left(\partial_{c} \hat{\psi}-\hat{\mu}\right) \varphi+\sum\left(D_{p} \hat{\psi}\right) \cdot \nabla^{p} \varphi\right\}=\int_{\Omega}\left\{\partial_{c} \hat{\psi}-\hat{\mu}+\sum(-1)^{p} \operatorname{div}^{p}\left(D_{p} \hat{\psi}\right)\right\} \varphi .
$$

This implies (5.3), since $\varphi$ is arbitrary.

Let $M$ denote the maximum of the grades of the response functions, and for any integer $p, 1 \leq p \leq M$, let

$$
\mathbf{L}_{p}=\hat{\boldsymbol{\zeta}} \otimes D_{p} \hat{\pi} \quad\left(\mathbf{L}_{0}=\hat{\boldsymbol{\xi}}\right) .
$$

Then

$$
\dot{\pi} \boldsymbol{\zeta}=\mathbf{L}_{0} \dot{c}+\sum \mathbf{L}_{p} \nabla^{p} \dot{c}
$$

(the sum being from $p=1$ to $p=M$ ).

The equation obtained upon substituting the constitutive equations into (5.1) is linear in the rates $\nabla^{p} \dot{c}, 0 \leq p \leq M+1$. We may therefore use (ii) of the Variation Lemma to conclude that the coefficient of each $\nabla^{p} \dot{c}$ must vanish, and this yields the relations

$$
\begin{aligned}
\partial_{c} \hat{\psi} & =\hat{\mu}+\operatorname{div} \mathbf{L}_{0}, \\
D_{p} \hat{\psi} & =\operatorname{sym}_{p-1}+\operatorname{div} \mathbf{L}_{p}, \quad 1 \leq p \leq M, \\
0 & =\operatorname{sym}_{M} .
\end{aligned}
$$


LEMMA 4. $\hat{\zeta}$ cannot vanish on an open set. Further,

$$
\text { Grade } \hat{\mu}=2 m, \quad \text { Grade } \hat{\pi}=m-1 \text { (strongly). }
$$

Proof. If $\hat{\zeta}$ vanished on an open set, then, by (5.6), $D_{m} \hat{\psi}$ would vanish on an open set, which contradicts $(\mathbf{F H})$.

Next, the term on the right side of (5.3) of highest (possible) order is

$$
D_{m m} \hat{\psi}(c, \mathbf{d}) \cdot \nabla^{2 m} c .
$$

By (3.1) and Lemma 1(iii) (considering $D_{m m} \hat{\psi}(c, 0)$ as a tensor of order $2 m$ ),

$$
\operatorname{sym}\left[D_{m m} \hat{\psi}(c, 0)\right] \neq \mathbf{0} .
$$

Thus (5.8) is not identically zero and, by (5.3), Grade $\hat{\mu}=2 \mathrm{~m}$.

It is clear from (5.6) and Lemma 1(ii) that $\mathbf{L}_{p}=\mathbf{0}$ for $p \geq m$; thus, in view of (5.5), Lemma 1(ii), and the fact that $\hat{\zeta}$ cannot vanish on an open set,

$$
\begin{aligned}
D_{p} \hat{\pi} & =\mathbf{0} \text { for } p \geq m, \\
D_{m} \hat{\psi} & =\operatorname{sym}\left(\hat{\zeta} \otimes D_{m-1} \hat{\pi}\right) .
\end{aligned}
$$

On the other hand, $D_{m-1} \hat{\pi}$ cannot vanish on an open set $\Sigma$, for if it did $(5.9)_{2}$ would yield $D_{m} \hat{\psi}=\mathbf{0}$ on $\Sigma$, and $\hat{\psi}$ would not be strongly of grade $m$. Hence Grade $\hat{\pi}=m-1$ (strongly).

LemMA 5. Grade $\hat{\psi}=1$, Grade $\hat{\mu}=2$, Grade $\hat{\pi}=0$.

Proof. In view of Lemma 4, it suffices to show that $m \geq 2$ leads to a contradiction. Assume that $m \geq 2$. Let

$$
\mathbf{P}=D_{m-1} \hat{\pi}
$$

Then, by $(5.9)_{2}, m D_{m} \hat{\psi}$ has components

$$
P_{j k \cdots a s} \zeta_{i}+P_{i k \cdots a s} \zeta_{j}+\cdots+P_{j k \cdots i s} \zeta_{a}+P_{j k \cdots a i} \zeta_{s} .
$$

Let $\mathbf{R}=\nabla^{r} c$ with $r \geq m+1$. Then $\partial_{\mathbf{R}} \hat{\psi}=\partial_{\mathbf{R}} \hat{\pi}=\mathbf{0}$ and

$$
P_{j k \cdots a s} \partial_{\mathbf{R}} \zeta_{i}+P_{i k \cdots a s} \partial_{\mathbf{R}} \zeta_{j}+\cdots+P_{j k \cdots i s} \partial_{\mathbf{R}} \zeta_{a}+P_{j k \cdots a i} \partial_{\mathbf{R}} \zeta_{s}=\mathbf{0} ;
$$

and if we take the inner product of this relation with $P_{j k \cdots a s} \partial_{\mathbf{R}} \zeta_{i}$ and sum over the indices $j k \cdots a s i$, we find that $\left|D_{m-1} \hat{\pi}\right|^{2}\left|\partial_{\mathrm{R}} \zeta\right|^{2}$ plus a sum of nonnegative terms must vanish. Therefore $\left|D_{m-1} \hat{\pi}\right|\left|\partial_{\mathbf{R}} \zeta\right|=0$, and, since $\mathbf{R}=\nabla^{r} c$ with $r \geq m+1$ is arbitrary,

$$
\left|D_{m-1} \hat{\pi}\right|\left|D_{r} \zeta\right|=0 \text { for } r \geq m+1 \text {. }
$$

But $\hat{\pi}$ is strongly of grade $m-1$; hence $D_{m-1} \hat{\pi}$ cannot vanish on an open set and

$$
D_{r} \zeta=0 \text { for } r \geq m+1 \text {. }
$$

Thus, by $(5.5)$ and $(5.7)_{2}$,

$$
D_{r}\left(\operatorname{div} \mathbf{L}_{0}\right)=0 \text { for } r \geq m+2,
$$

and, since Grade $\hat{\psi}=m,(5.6)_{1}$ yields

$$
D_{r} \hat{\mu}=0 \text { for } r \geq m+2 .
$$

Moreover, since $m \geq 2$, it follows that $2 m \geq m+2$, and $D_{2 m} \hat{\mu}=0$, which contradicts $(5.7)_{1}$. 
LEMMA 6. The restrictions (3.4) are satisfied and Grade $\hat{\boldsymbol{\zeta}}=$ Grade $\hat{\boldsymbol{\xi}}=1$.

Proof. In view of Lemma 5, (5.6) reduce to (3.4). Since Grade $\hat{\psi}=1,(3.4)_{2}$ yields Grade $\hat{\boldsymbol{\xi}} \leq 1$. But by (3.1), $\partial_{\mathrm{gg}} \hat{\psi}$ cannot be identically zero; hence Grade $\hat{\boldsymbol{\xi}}=1$. Since Grade $\hat{\pi}=0$, this and (3.2) imply that Grade $\hat{\zeta}=1$.

Lemmas 5 and 6 yield all of the assertions in (i) and (ii). To verify (iii), note that, if $\partial_{c} \hat{\pi}(c)=0$ at a particular $c$, then (3.2) and $(3.4)_{2}$ would render $\hat{\psi}(c, \mathbf{g})$ independent of $\mathbf{g}$ at that $c$, which would violate $(3.1)_{3}$.

Finally, assertion (iv) is a direct consequence of (5.2).

This completes the proof of the Compatibility Theorem.

IV. Quasi-linear theory.

6. Consequences of the second law. The results of the Compatibility Theorem take a particularly simple form when the constitutive equations are quasi linear in the sense of the hypothesis:

For each $c, \hat{\mu}(c, \mathbf{g}, \mathbf{G}), \hat{\mathbf{h}}(c, \mathbf{g}, \mathbf{G}, \mathbf{H})$, and $\hat{\boldsymbol{\xi}}(c, \mathbf{g})$ are affine

functions of their remaining arguments.

Compatibility TheORem for QuAsi-Linear Response. Assume that the constitutive equations are compatible with thermodynamics, and that assumptions (FH) and (QL) are satisfied. Then the constitutive equations have the specific form:

$$
\begin{aligned}
\psi & =\psi_{0}(c)+\frac{1}{2} \mathbf{g} \cdot \mathbf{A g}, \\
\mu & =\psi_{0}^{\prime}(c)-\mathbf{A} \cdot \mathbf{G}, \\
\boldsymbol{\xi} & =\mathbf{A g}, \\
\mathbf{h} & =-\mathbf{K}(c) \nabla \mu,
\end{aligned}
$$

where $\mathbf{A}$ and $\mathbf{K}(c)$ (for each $c$ ) are second-order tensors with

$$
\text { A symmetric, } \quad \mathbf{A} \geq 0, \quad \mathbf{A} \neq 0, \quad \mathbf{K}(c) \geq 0 .
$$

The proof of this theorem is greatly facilitated by

Lemma 7. Let $U$ and $V$ be vector spaces, let $F, G: U \rightarrow V$ be linear transformations with $G$ surjective and such that

$$
G u \cdot F u \geq 0
$$

for all $u \in U$. Then there is a linear transformation $K: V \rightarrow V$ with $K \geq 0$ such that

$$
F=K G \text {. }
$$

Proof. Our first step is to show that

$$
G u=0 \text { implies } F u=0 .
$$

Assume $G u=0$. Then (6.3) implies that $\varphi(w)=G w \cdot F w$ has a minimum at $w=u$; thus, since $G$ and $F$ are linear, if we expand $\varphi(w)$ with $w=u+z$ we find that

$$
G z \cdot F u=0 \text { for every } z \in U \text {. }
$$

Since $G$ is surjective, this yields $F u=0$. Thus $(6.5)$ is satisfied. 
Next, in view of the surjectivity of $G$, for each $v \in V$ there is a (not necessarily unique) $u(v) \in U$ such that $G u(v)=v$. We define $K: V \rightarrow V$ by $K v=F u(v)$. Choose $p \in U$ and let $v=G p$. Then $K G p=F u(v)$ and $G u(v)=v=G p$. Since $F$ and $G$ are linear, (6.5) implies that $F u(v)=F p$. Hence $K G p=F p$ and (6.4) is valid.

We have only to establish the linearity and positivity of $K$. Using the linearity of $G$ and the definition of $u$, it is not difficult to verify that the composition $G \circ u$ is linear; by virtue of (6.5), this implies that $K=F \circ u$ is linear.

Finally, (6.3), (6.4), and the surjectivity of $G$ imply that $K \geq 0$.

Proof (Theorem). By hypothesis, $\hat{\boldsymbol{\xi}}(c, \mathbf{g})$ is an affine function of $\mathbf{g}$. We may therefore use (3.1) and $(3.4)_{2}$ to conclude that

$$
\hat{\psi}(c, \mathbf{g})=\psi_{0}(c)+\frac{1}{2} \mathbf{g} \cdot \mathbf{A}(c) \mathbf{g}
$$

with $\mathbf{A}(c)$ symmetric, $\mathbf{A}(c) \geq 0, \mathbf{A}(c) \neq 0$. Thus $(3.4)_{2}$ implies that

$$
\hat{\boldsymbol{\xi}}(c, \mathbf{g})=\mathbf{A}(c) \mathbf{g}
$$

while $(3.4)_{1}$ yields

$$
\hat{\mu}(c, \mathbf{g}, \mathbf{G})=\psi_{0}^{\prime}(c)-\frac{1}{2} \mathbf{g} \cdot \mathbf{A}^{\prime}(c) \mathbf{g}-\mathbf{A}(c) \cdot \mathbf{G} ;
$$

since $\hat{\mu}(c, \mathbf{g}, \mathbf{G})$ is affine in $(\mathbf{g}, \mathbf{G}), \mathbf{A}^{\prime}=0$ and

$$
\hat{\mu}(c, \mathbf{g}, \mathbf{G})=\psi_{0}^{\prime}(c)-\mathbf{A} \cdot \mathbf{G} .
$$

We have established $(6.1)_{1}-(6.1)_{3}$ and that part of (6.2) concerning $A$; to complete the proof we have only to verify the results concerning the mass flux.

Let $\boldsymbol{\omega}=(\mathbf{g}, \mathbf{G}, \mathbf{H}), \mathbf{u}=\nabla \hat{\mu}$, and for convenience suppress the argument $c$. Then, in view of (3.5),

$$
\hat{\mathbf{h}}(\boldsymbol{\omega}) \cdot \mathbf{u}(\boldsymbol{\omega}) \leq 0,
$$

where $\hat{\mathbf{h}}$ and $\mathbf{u}$ are mappings of $W=\mathbb{R}^{3} \times S_{2} \times S_{3}$ into $\mathbb{R}^{3}$, and (6.8) holds for all $\boldsymbol{\omega} \in W$.

Our next step will be to show that

$\mathbf{u}$ is surjective.

By $(6.1)_{2}$,

$$
\mathbf{u}(\boldsymbol{\omega})=\psi_{0}^{\prime \prime} \mathbf{g}-\mathbf{A H},
$$

where $\mathbf{A H}$ is the vector with components

$$
(\mathbf{A H})_{k}=A_{i j} H_{i j k} .
$$

To verify (6.9) it therefore suffices to show that given any vector $v \in \mathbf{R}^{3}$, there is an $H \in S_{3}$ such that (in components)

$$
A_{i j} H_{i j k}=v_{k} .
$$

Since $\mathbf{A}$ is symmetric and $\mathbf{A} \neq \mathbf{0}$, we may assume, without loss in generality, that $\mathbf{A}$ is diagonal with $A_{11} \neq 0$. Then $\mathbf{H}$ with all components zero except

$$
H_{11 k}=H_{1 k 1}=H_{k 11}=v_{k} / A_{11} \quad(k=1,2,3)
$$

is a solution of $(6.10)$. 
Note that, since $\mathbf{u}$ is linear and surjective, (6.8) can hold for all $\boldsymbol{\omega}$ only if $\hat{\mathbf{h}}(\mathbf{0})=\mathbf{0}$; hence $\hat{\mathbf{h}}$ is also linear.

We have only to establish the relation $(6.1)_{4}$ with $\mathbf{K}=\mathbf{K}(c) \geq 0$; but this is an immediate consequence of (6.8), (6.9), and Lemma 7 with $G=\hat{\mathbf{u}}, F=-\hat{\mathbf{h}}, U=W$, and $V=\mathrm{R}^{3}$.

For an isotropic material (6.1) simplify considerably. Indeed, there are scalars $a$ and $k(c)$ such that

$$
\begin{array}{ll}
\mathbf{A}=a \mathbf{1}, & \mathbf{K}(c)=k(c) \mathbf{1}, \\
a>0, & k(c) \geq 0,
\end{array}
$$

with 1 the identity tensor, and (6.1) reduces to

$$
\begin{aligned}
\psi & =\psi_{0}(c)+\frac{1}{2} a|\mathbf{g}|^{2}, \\
\mu & =\psi_{0}^{\prime}(c)-a \Delta c, \\
\boldsymbol{\xi} & =a \mathbf{g}, \\
\mathbf{h} & =-k(c) \nabla \mu,
\end{aligned}
$$

with $\Delta$ the Laplacian. We call $a$ the capillarity constant, $k(c)$ the diffusivity.

7. The Cahn-Hilliard equation. Boundary-value problems. The general anisotropic constitutive equations (6.1), when combined with balance of mass $(1.3)_{1}$ reduce to a single partial differential equation for the concentration:

$$
\dot{c}=\operatorname{div}\left\{\mathbf{K}(c) \nabla\left[\psi_{0}^{\prime}(c)-\mathbf{A} \cdot \nabla \nabla c\right]\right\}+q .
$$

For an isotropic material with constant diffusivity this equation has the particularly simple form:

$$
\dot{c}=k \Delta\left[\psi_{0}^{\prime}(c)-a \Delta c\right]+q,
$$

which is the Cahn-Hilliard equation.

Consider now the more general differential equation (7.1), supplemented by the constitutive equations:

$$
\begin{aligned}
& \psi=\psi_{0}(c)+\frac{1}{2} \nabla c \cdot \mathbf{A} \nabla c \\
& \boldsymbol{\xi}=\mathbf{A} \nabla c \\
& \mu=\psi_{0}^{\prime}(c)-\mathbf{A} \cdot \nabla \nabla c \\
& \mathbf{h}=-\mathbf{K}(c) \nabla \mu .
\end{aligned}
$$

We shall consider the boundary conditions (1.5) and (1.6), but with $c$ as capillarity potential and $\boldsymbol{\xi}$ as capillarity vector:

(i) isolated boundary:

$$
\boldsymbol{\xi} \cdot \mathbf{n}=0 \quad \text { and } \quad \mathbf{h} \cdot \mathbf{n}=0 \quad \text { on } \partial \Omega
$$

(ii) uniform boundary:

$$
c=c_{b} \text { and } \mu=\mu_{b} \text { on } \partial \Omega,
$$

with $c_{b}$ and $\mu_{b}$ constant.

We define a Gibbs function for the uniform boundary through

$$
G(c)=\psi_{0}(c)-\mu_{b} c
$$

then, as a direct consequence of (1.7), (1.8), and $(7.3)_{1}$, we have the 
Growth Theorem For Quasi-Linear Response. Let $c$ be a solution of the differential equation (7.1) with $q=0$. Then:

(i) for an isolated boundary

$$
\begin{gathered}
\frac{d}{d t} \int_{\Omega} c=0, \\
\frac{d}{d t} \int_{\Omega}\left\{\psi_{0}(c)+\frac{1}{2} \nabla c \cdot \mathbf{A} \nabla c\right\} \leq 0
\end{gathered}
$$

(ii) for a uniform boundary

$$
\frac{d}{d t} \int_{\Omega}\left\{G(c)+\frac{1}{2} \nabla c \cdot \mathbf{A} \nabla c\right\} \leq 0 .
$$

Remark. The uniform boundary defined by (7.5) is in equilibrium if

$$
\mu_{b}=\psi_{0}^{\prime}\left(c_{b}\right)
$$

this condition allows us to rewrite (7.5) as

$$
c=c_{b} \text { and } \mathbf{A} \cdot \nabla \nabla c=0 \text { on } \partial \Omega \text {. }
$$

For an isotropic material (cf. (6.11)) the condition for an isolated boundary reduces to

$$
\partial c / \partial n=0 \text { and } \partial(\Delta c) / \partial n=0 \text { on } \partial \Omega,
$$

where $\partial / \partial n$ denotes the normal derivative on $\partial \Omega$. On the other hand, for a uniform boundary isotropy yields the condition

$$
c=c_{b} \quad \text { and } \quad \psi_{0}^{\prime}(c)-a \Delta c=\mu_{b} \quad \text { on } \partial \Omega,
$$

or equivalently, if the boundary is in equilibrium,

$$
c=c_{b} \quad \text { and } \quad \Delta c=0 \text { on } \partial \Omega .
$$

Appropriate initial/boundary-value problems ${ }^{11}$ consist of the partial differential equation (7.1) on $\Omega \times(0, \infty)$, the boundary condition (7.4) or (7.5) on $\partial \Omega \times(0, \infty)$, and the initial condition

$$
c(\mathbf{x}, 0)=C(\mathbf{x}) \text { on } \Omega,
$$

where $C(\mathbf{x})$ is the prescribed initial concentration.

Remark. More generally one might consider as boundary conditions the prescription of either $\mu$ or $\mathbf{h} \cdot \mathbf{n}$ and either $c$ or $\boldsymbol{\xi} \cdot \mathbf{n}$ at each point of $\partial \Omega$.

Acknowledgment. This work was supported by the Army Research Office and by the National Science Foundation.

\footnotetext{
"For the isotropic problem (7.2), (7.11), and (7.14), von Wahl [19] proves existence under reasonable growth conditions on $\psi_{0}$, while Elliott and Songmu [20] establish existence and uniqueness in one spacedimension for $\psi_{0}$, the usual double-well potential. For related results and discussion concerning this problem, cf. Novick-Cohen and Segel [22], Novick-Cohen [17], Nicolaenko and Scheurer [16], Songmu [23], and Elliott and French [24]. To my knowledge there are no results for a uniform boundary.
} 


\section{REFERENCES}

[1] J. W. Gibbs, On the equilibrium of heterogeneous substances, Trans. Connecticut Acad. 3, 108-248 (1876), 343-524 (1878). Reprinted in: The Scientific Papers of J. Willard Gibbs, Vol. 1, Dover, New York, 1961

[2] J. D. van der Waals, The thermodynamic theory of capillarity under the hypothesis of a continuous variation of density (in Dutch), Verhandel. Konink. Akad. Weten. Amsterdam (Sec. 1) Vol. 1, No. 8 (1893)

[3] D. J. Korteweg, Sur la forme que prennent les équations des mouvement des fluides si l'on tientcomple des forces capillaires par des variations de densité, Arch. Neerl. Sci. Exactes Nat. Ser. 11, 6, 1-24 (1901)

[4] J. W. Cahn and J. E. Hilliard, Free energy of a nonuniform system. I. Interfacial free energy, J. Chem. Phys. 28, 258-267 (1958)

[5] L. D. Landau and E. M. Lifschitz, Electrodynamics of Continuous Bodies, Pergamon, New York, 1960

[6] B. D. Coleman and W. Noll, The thermodynamics of elastic materials with heat conduction and viscosity, Arch. Rat. Mech. Anal. 13, 167-178 (1963)

[7] J. W. Cahn, On spinodal decomposition, Acta Metallurgica 9, 795-801 (1961)

[8] J. W. Cahn, On spinodal decomposition in cubic crystals, Acta Metallurgica 10, 179-183 (1962)

[9] J. W. Cahn and J. E. Hilliard, Spinodal decomposition: a reprise, Acta Metallurgica 19, 151-161 (1971)

[10] D. W. Hoffman and J. W. Cahn, $A$ vector thermodynamics for anisotropic surfaces-1. Fundamentals and applications to plane surface junctions, Surface Sci. 31, 368-388 (1972)

[11] J. W. Cahn and D. W. Hoffman, $A$ vector thermodynamics for anisotropic surfaces-2. Curved and faceted surfaces, Acta Metallurgica 22, 1205-1214 (1974)

[12] B. Widom, Structure and thermodynamics of interfaces, in Statistical Mechanics and Statistical Methods in Theory and Application, Plenum, New York, 1977

[13] J. Fernandez-Diaz and W. O. Williams, A generalized Stefan condition, Zeit. Angew. Math. Phys. 30, 749-755 (1979)

[14] M. E. Gurtin, Toward a nonequilibrium thermodynamics of two phase materials, Arch. Rat. Mech. Anal. 100, 275-312 (1988)

[15] J. E. Dunn and J. Serrin, On the thermomechanics of interstitial working, Arch. Rat. Mech. Anal. 88, 95-133 (1985)

[16] B. Nicolaenko and B. Scheurer, Low-dimensional behavior of the pattern formation Cahn-Hilliard equation, in Trends in the Theory and Practice of Non-Linear Analysis, V. Lakschmikantham (ed.), Elsevier Science, New York, 1985

[17] A. Novick-Cohen, Energy methods for the Cahn-Hilliard equation, IMA Preprint 157, Institute for Mathematics and its Applications, University of Minnesota, 1985

[18] J. A. Serrin, Continuum model for chemical mixture dynamics, Developments in Mechanics 13, Appl. Mech. Div., Am. Soc. Mech. Eng., 1985

[19] W. von Wahl, On the Cahn-Hilliard equation $u^{\prime}+\Delta^{2} u-\Delta f(u)=0$, Delft Progress Report 10, 291-310, Delft University, The Netherlands, 1985

[20] C. M. Elliott and Z. Songmu, On the Cahn-Hilliard equation, Arch. Rat. Mech. Anal. 96, 339-357 (1986)

[21] M. E. Gurtin, On the two-phase Stefan problem with interfacial energy and entropy, Arch. Rat. Mech. Anal. 96, 199-241 (1986)

[22] A. Novick-Cohen and L. A. Segel, Nonlinear aspects of the Cahn-Hilliard equation, Physica (D) 10, 277-298 (1986)

[23] Z. Songmu, Asymptotic behavior of the solution to the Cahn-Hilliard equation, Technical Report 25, Center for Applied Mathematics, Purdue University, 1986

[24] C. M. Elliott and D. A. French, Numerical studies of the Cahn-Hilliard equation for phase separation, IMA J. Appl. Math. 38, 97-128 (1987) 